\title{
Le phosphore assimilable des sols : sa représentation par un modèle fonctionnel à plusieurs compartiments
}

\author{
JC Fardeau \\ DPVE, CE Cadarache, 13108 Saint-Paul-lez-Durance, France
}

(Reçu le 11 mai 1992; accepté le 20 février 1993)

\begin{abstract}
Résumé - L'analyse compartimentale des cinétiques d'échange isotopique des ions phosphate permet de démontrer que le phosphore $(\mathrm{P})$ biodisponible des sols est un ensemble hétérogène contenant des ions phosphate liés à la matrice du sol, mais susceptibles de gagner la solution du sol en des temps très variables. Le $\mathrm{P}$ biodisponible est donc un système à plusieurs compartiments. En partant de considérations sur les conditions de fonctionnement des racines et des systèmes racinaires, le $\mathrm{P}$ assimilable a été découpé en 5 compartiments différenciés par la durée nécessaire à leur transfert dans la solution du sol. Le pool central, $\mathrm{P}_{\mathrm{L}}$, est celui des ions libres pour lesquels le temps de transfert de la phase solide à la solution du sol est inférieur à $15 \mathrm{~s}$. Les 4 autres pools, B, C, D et F, sont tous directement reliés au pool central. Ils contiennent les ions dont le temps de transfert vers la solution du sol est compris, dans ce système en état stationnaire, entre $1 \mathrm{~m}$ et $1 \mathrm{j}$ (pool B), $1 \mathrm{j}$ et 3 mois (pool C), 3 mois et 1 an (pool D) et est supérieur à 1 an pour le pool $\mathrm{F}$. Ce modèle ne prend pas en considération le $\mathrm{P}$ organique dont la transformation vers la forme biodisponible suit des processus différents. Pour favoriser la comparaison visuelle rapide des caractéristiques essentielles du $\mathrm{P}$ biodisponible présent dans les échantillons issus de prélèvements différents, une représentation schématique de ce modèle global a été mise au point. À titre d'exemple, on montre que des prélèvements répétitifs ou des fertilisations excédentaires de phosphore modifient tous les compartiments. Cette représentation permet d'illustrer l'existence de 2 types de pouvoir tampon du sol pour les ions phosphate : l'un est qualifié de apouvoir tampon instantané», l'autre de «pouvoir tampon retard»; ce dernier assure le renouvellement des ions phosphate dans la solution en fonction du temps et de la quantité prélevée ou ajoutée.
\end{abstract}

phosphore biodisponible / compartiment / modèle fonctionnel / pouvoir tampon

Summary - Available soil phosphate: its representation by a functional multiple compartment model. Analysis of the isotopic exchange kinetics of phosphate ions which occurs in a soil-solution system maintained in steadystate shows that the available soil phosphate is a heterogeneous system that lends itself to compartmentalization. Available soil $P$ has been subdivided into 5 pools in relation to the functioning of roots and root systems. The central and most important pool for $P$ uptake by crops is the pool $P_{L}$ of free phosphate ions: this pool contains ions which can be transferred into the soil solution in $<15 \mathrm{~s}$. The 4 other pools, B, C, D and F, branch directly off the central pool. In this system studied in steady-state, the pool $B$ contains ions which can exchange with the phosphate ions of the soil solution in between $1 \mathrm{~min}$ and $1 \mathrm{~d}$. The pool $C$ contains the ions able to enter the solution in steady-state between $1 \mathrm{~d}$ and 3 months. The pool $D$ contains ions which can exchange with phosphate ions in the solution in 3 months to $1 \mathrm{yr}$. The pool $F$ contains phosphate ions exchangeable after $>1 \mathrm{yr}$. This model concerns only inorganic $P$, organic $P$ following other rules of transformation, such as those of microbial mineralization. A schematic functional model has been developed to summarize this information. The model applied to various soil samples taken from 2 long-term field experiments showed that repeated fertilization or exhaustive cropping can modify all the pools. This scheme shows 2 kinds of phosphate buffering capacity $(P B C)$ for soils. The first kind, which depends on the $P_{L}$ pool, is called instantaneous $P B C$ '; the second kind, which depends on the 4 other pools, is called 'delayed PBC': its effect on the maintenance of phosphate ion concentration in the soil solution is time-dependent.

available phosphate / conceptual model / pool / buffering capacity 


\section{INTRODUCTION}

Le phosphore présent dans les sols n'est pas un ensemble homogène. Le concept de phosphore actif vis-à-vis des plantes, et son corollaire de phosphore dormant, a été décrit pour la première fois il y a déjà plus d'un siècle (Daubeny, 1845). Cette démarche conduit à représenter le phosphore du sol par un modèle à 2 compartiments. La mise en évidence des différents compartiments d'un système complexe, puis la description de leur organisation spatiale, sont généralement réalisables en faisant appel à des techniques de traçage isotopique (Sheppard, 1962; Shipley and Clark, 1972; Atkins, 1973).

Les premières expériences culturales réalisées en présence de phosphates marqués par du 32P (Larsen, 1952; Fried and Dean, 1952) semblaient confirmer l'analyse bicompartimentale du phosphore du sol : la fraction biodisponible du phosphore du sol était alors considérée comme un compartiment homogène contenant les quantités L (Larsen, 1952) ou A (Fried and Dean, 1952) de phosphore. Des agronomes ont alors continué de chercher à quantifier le phosphore biodisponible d'un sol en utilisant la méthode de dilution isotopique qui n'est opérationnelle, en théorie (Sheppard, 1962), que dans un système à un seul compartiment. Dans le même temps, des physico-chimistes démontraient, en injectant des ions ${ }^{32} \mathrm{PO}_{4}$ dans des systèmes solsolution en état stationnaire, d'une part que des échanges isotopiques se produisaient entre les ions phosphate présents dans la solution du sol et d'autres ions phosphate associés à la phase solide, et d'autre part que ces échanges n'étaient pas instantanés (Wiklander, 1950; Barbier et Tyskiewicz, 1952, 1954). L'existence des cinétiques d'échanges iso-ioniques permettait à d'autres auteurs de conclure que les ions phosphate isotopiquement échangeables d'un système sol-solution de sol étaient localisés dans plusieurs compartiments (Talibudeen, 1958; Lamm, 1962; Ulrich et al, 1962; Probert and Larsen, 1972; Kotze and Diest, 1973). Mais cette conclusion est restée théorique (Triboï et Gachon, 1988) : aucune organisation spatiotemporelle des différents compartiments du système n'était proposée. Seul un des compartiments, celui du pool des ions libres directement assimilables par les plantes, était décrit avec précision (Fardeau et Marini, 1968; Salcedo et al, 1991); l'ensemble des autres ions phosphate isotopiquement échangeables restaient logés dans une "boîte noire» (Fardeau et al, 1991).
En associant des résultats expérimentaux provenant d'expériences de cinétiques d'échanges isotopiques dans des systèmes sol-solution et de cultures en pots en présence d'ions ${ }^{32} \mathrm{PO}_{4}$, il a été possible de démontrer l'identité physique des ions phosphate isotopiquement échangeables et des ions biodisponibles (Fardeau et Jappé, 1976). Cette observation était confirmée par la suite même pour les plantes comportant des endomycorhizes qui leur sont parfois associées (Gianinazzi-Pearson et al, 1981). Ces résultats permettent d'affirmer :

- que le phosphore biodisponible est un ensemble pluricompartimental dans lequel il existe des compartiments de mobilité, donc de biodisponibilité très diverse; ce faisant ils illustrent l'existence d'une contradiction entre les conclusions déduites des méthodes du type valeur $L$ ou valeur $A$ qui ont abouti à donner du phosphore biodisponible l'image d'un ensemble homogène et les conclusions déduites des cinétiques d'échanges isotopiques qui obligent à considérer le phosphore biodisponible comme un ensemble constitué de nombreux compartiments;

- qu'il faut et suffit de décrire l'organisation compartimentale de l'ensemble des ions phosphate isotopiquement échangeables d'un système solsolution pour décrire le phosphore biodisponible d'un sol.

L'objet du présent travail est :

- de résoudre la contradiction existant entre les conclusions déduites de l'utilisation des méthodes de type $A$ ou $L$ et celles déduites de l'analyse des cinétiques d'échanges isotopiques;

- de présenter une méthode permettant de quantifier, au sein du phosphore biodisponible, des compartiments fonctionnels sans avoir à les extraire;

- de proposer un modèle susceptible de rendre compte et de schématiser l'organisation spatiale des différents compartiments représentatifs du phosphore biodisponible du sol;

- enfin d'illustrer, au moyen d'exemples agronomiques, l'intérêt du modèle.

\section{MATÉRIEL ET MÉTHODES}

\section{Définition d'un compartiment ou pool}

Un compartiment, ou pool, est la quantité d'une substance donnée dont toutes les parties constitutives 
possèdent une cinétique identique de transport ou de transformation (Sheppard, 1962; Atkins, 1973); il s'agit donc d'un ensemble homogène au regard d'un processus cinétique donné. Cette définition montre que la notion de compartiment est dépendante du choix du pas de temps retenu entre 2 observations successives; il pourra en résulter un certain arbitraire dans la définition même d'un compartiment.

\section{Méthode de l'analyse compartimentale}

Cette méthode peut être utilisée chaque fois que l'objectif d'une recherche est d'aboutir à la description des relations spatio-temporelles existant entre les différents pools d'un système complexe. Les transferts de traceurs au sein d'un système complexe étant identiques à ceux de l'élément tracé, l'analyse compartimentale consiste à injecter un traceur dans un compartiment donné, puis à suivre d'une part la cinétique de son départ du compartiment où il a été introduit et, d'autre part, si possible, la cinétique de son arrivée dans d'autres compartiments. L'analyse mathématique de ces cinétiques est facilitée lorsque les systèmes étudiés sont en état stationnaire, ou équilibre dynamique, durant la période choisie pour déterminer les cinétiques. Dans cet état, et dans cet état seulement, la cinétique de transfert d'une substance depuis un compartiment jusqu'à un second est une cinétique du premier ordre dont la fonction représentative est une exponentielle, alors que celle du transfert depuis un compartiment jusqu'à plusieurs autres est représentée par une fonction somme d'exponentielles (Shipley, 1972; Atkins, 1973). C'est pourquoi le nombre de pools présents dans le système est alors égal au nombre d'exponentielles obtenues plus 1. Enfin, l'organisation spatiale des compartiments peut être soit de type linéaire où, à l'image d'un train, l'entrée dans un compartiment donné nécessite d'accéder préalablement dans le compartiment qui le précède, soit de type mamellaire où tous les compartiments sont accessibles à partir d'un compartiment unique jouant un rôle central et déterminant dans le fonctionnement et l'équilibre du système (Sheppard, 1972). II est également possible de rencontrer des types mixtes; cependant ces derniers types seront assimilés à l'un ou l'autre modèle selon que la liaison entre le compartiment d'injection du traceur et les autres compartiments du système se fera par un seul ou plusieurs compartiments. Les variables utilisées pour décrire la méthode puis le modèle ont été regroupées dans le tableau I.

\section{Adaptation de la méthode à l'établissement de cinétiques d'échanges isotopiques des ions phosphate dans des systèmes sol-solution}

La méthode a été amplement décrite précédemment (Fardeau et al, 1991). Les cinétiques sont réalisées

Tableau I. Liste des variables utilisées.

Variables

Symbole Signification Unités usuelles

A

$B, C, D, F$

$C_{P}$

$E_{P i e 1}$

$E_{\text {Piet }}$

$F_{m}$

$K_{m}$

$L$

$n$

$P_{M T}$

$P_{T}$

$P_{L}$

$P_{L} / C_{P}$

$P_{S}$

R

$r_{1}$

$r_{t}$

$t$

$T_{m}$
Quantité de $\mathrm{P}$ biodisponible selon Fried

Pools de $P$ biodisponible

Concentration du $\mathrm{P}$ dans la solution du sol

Quantité de $P$ isot échangeable en $1 \mathrm{mn}$

Quantité de $P$ isot échangeable en $t \mathrm{mn}$

Flux moyen d'échange entre sol et solution

Constante moyenne de vitesse d'échange

Quantité de $\mathrm{P}$ biodisponible selon Larsen

Coefficient de la régression $\log r_{t}-\log t$

Phosphore minéral total d'un échantillon

Phosphore total d'un échantillon

Quantité de $P$ isot échangeable dans le pool $P_{L}$

Volume apparent de distribution du pool $P_{L}$

Quantité de phosphore en solution. $P_{S}=10 C_{P}$

Radioactivité totale injectée dans le mélange

Radioactivité en solution 1 min après l'injection

Radioactivité en solution $t$ min après l'injection

Temps après l'injection du ${ }^{32} \mathrm{P}$

Temps moyen de séjour des ions $\mathrm{PO}_{4}$ en solution
$\operatorname{mgP}(\mathrm{kg} \mathrm{sol})^{-1}$

mgP.l-1
mgP (kg sol)-1
mgP (kg sol)-1
mgP (kg sol $)^{-1} \mathrm{mn}^{-1}$
$\mathrm{mn}^{-1}$
$\mathrm{mgP}(\mathrm{kg} \mathrm{sol})^{-1}$
sans unité
$\mathrm{mgP}(\mathrm{kg} \mathrm{sol})^{-1}$
$\mathrm{mgP}(\mathrm{kg} \mathrm{sol})^{-1}$
$\mathrm{mgP}(\mathrm{kg} \mathrm{sol})^{-1}$
$\mathrm{l}(\mathrm{kg} \mathrm{sol})^{-1}$
$\mathrm{mgP}(10 \mathrm{ml})^{-1}$
$\mathrm{~Bq}$
$\mathrm{~Bq}$
$\mathrm{~Bq}$
$\mathrm{~min}$
$\mathrm{~min}$


dans des systèmes sol-solution de rapport $1 / 10$ en état stationnaire obtenu par une agitation du mélange pendant une nuit. Dans un tel système, les transferts de matière, quel que soit l'élément considéré, dans le sens solution-phase solide, sont égaux aux échanges inverses. L'important est d'injecter une quantité $\mathrm{R}$ de radioactivité, sous forme d'ions ${ }^{32} \mathrm{PO}_{4}$ sans entraîneur, sans perturber l'état stationnaire des systèmes sol-solution. Les prélèvements, effectués pour connaître d'une part la concentration $C_{P}$ du phosphore dans la solution et d'autre part les quantités, $r_{t}$, de radioactivité restant dans la solution en fonction du temps, sont filtrés sur des membranes à pores de $0,01 \mu \mathrm{m}$. L'utilisation de cette porosité permet de doser $C_{P}$ par colorimétrie (John, 1970) en évitant toute erreur analytique qui résulterait de la présence de particules de sol. Le système étant en état stationnaire, la valeur de $C_{\rho}$ est invariante durant la durée des expériences. Quel que soit le système solsolution de sol, c'est-à-dire quel que soit le sol, la relatin $r_{t} / R=f(t)$ représentative du transfert des ions phosphate depuis la solution vers la phase solide du sol, pour des durées d'échanges isotopiques comprises entre $30 \mathrm{~s}$ et 4 mois, est de la forme générale (Fardeau et al, 1985; Fardeau et al, 1991) :

$$
r_{t} / R=\left(r_{1} / R\right)\left[\mathrm{t}+\left(r_{1} / R\right)^{1 / m}\right]-n+r_{e d} / R
$$

La valeur de $r_{\mathrm{eq}} / R$ est déduite de la quantité, $P_{M T}$, de phosphore minéral total de l'échantillon. En effet, lorsque l'équilibre isotopique est atteint, la radioactivité spécifique (RAS), c'est-à-dire le rapport de la quantité de traceur à celle de l'élément tracé, est la même en tout point du système. C'est pourquoi la RAS, $r_{\mathrm{eq}}{ }^{\prime}$ $P_{s}$, du $P$ des ions phosphate présents dans la solution, $P_{s}$ étant la quantité de phosphore dans la solution du sol, est égale à celle, $R / P_{M T}$, de l'ensemble des ions phosphate présents dans le système. Puisque dans un mélange sol-solution de rapport $1 / 10$ la quantité $P_{s}=10 C_{P}, r_{\text {eq }} / R=10 C_{P} / P_{M T}$. La valeur de $r_{\mathrm{eq}} / R$ sera donc comprise entre 0,005 et 0,0005 pour la majorité des échantillons de terre. Les termes correctifs $\left(r_{1} / R\right)^{1 / n}$ et $r_{\text {eq }} / R$ n'influencent la valeur de $r_{t} / R$ de manière significative, pour des valeurs de $t$ comprises entre $1 \mathrm{~min}$ et 1 an, que pour des échantillons de terre à pouvoir fixateur très élevé (Fardeau et al, 1985).

Pour la majorité des échantillons de terre prélevés sur des sols cultivés (Wiklander, 1950; Fardeau et al, 1991) la formule précédente [1] peut être remplacée par la formule simplifiée [2] :

$$
r_{t} / R=\left(r_{1} / R\right) t^{n}
$$

La quantité, $E_{\text {Piet, }}$ de phosphore isotopiquement échangeable à l'instant $t$ est calculée en faisant l'hypothèse qu'à tout instant $t$ la $R A S, R / E_{\text {Piet }}$, de l'ensemble des ions phosphate isotopiquement échangeables est égale à celle, $r_{t} / P_{S}$, des ions phosphate présents dans la solution:

soit $\left(R / E_{\text {Piet }}\right)=\left(r_{R} / P_{S}\right)$ ou $E_{\text {Piet }}=10 C_{P}\left(R / r_{t}\right)$
L'association de l'équation [2], utilisable sous réserve, et de l'équation [3] conduit à :

$$
E_{\text {Piet }}=E_{\text {Pie1 }} \text { tr }
$$

$E_{P i e 1}$ étant la quantité de phosphore isotopiquement échangé durant la première minute d'échange. Enfin la détermination expérimentale des paramètres $r_{1} / R$ et $\mathrm{n}$ de l'équation [1] ainsi que celle de la valeur de $C_{P}$ permettent de calculer la constante de vitesse d'échange, $K_{m}=n /\left(r_{1} / R\right)^{1 / n}$, des ions phosphate entre phase solide et phase liquide, le temps moyen de séjour, $T_{m}=1 / K_{m}$, des ions phosphate dans la solution du sol et le flux moyen d'échange, $F_{m}=\left(P_{S}\right)\left(K_{m}\right)$ des ions entre phase solide et phase liquide (Fardeau et al, 1991).

\section{Technique de recherche du modèle d'organisation spatiale des différents compartiments dans un système complexe}

Une première méthode utilisable pour savoir si un système pluricompartimental est de type linéaire ou mamellaire consiste à déterminer les quantités de radioactivité transférées au cours du temps dans des compartiments autres que celui où le traceur a été injecté. Cette démarche impose d'extraire, par des méthodes physiques ou chimiques, tout ou partie de certains compartiments pour mesurer au cours du temps les variations de la RAS du phosphore qu'ils contiennent. Or les méthodes d'extraction perturbent généralement l'état stationnaire du système et la distribution du phosphore entre ses différentes formes (Fardeau et al, 1988) : elles rendent alors toute interprétation des résultats délicate lorsque les mécanismes réellement mis en jeu au cours des extractions ne sont pas connus (Fardeau et al, 1988). Néanmoins, une telle démarche, dans laquelle le traceur ${ }^{32} \mathrm{PO}_{4}$ et des extractions séquentielles plus ou moins sélectives, telle que celle de Chang et Jackson (1956), ont été utilisés simultanément, a permis de démontrer que n'importe quelle forme de phosphore minéral pouvait constituer une source de phosphore biodisponible pour les plantes (Jenkins, 1966; Halstead, 1967; Tandon and Kurtz, 1968; Gachon, 1972).

Une seconde méthode utilisable pour analyser la structure spatio-temporelle d'un système pluricompartimental, et qui est celle choisie dans notre étude, consiste à modifier la taille d'un compartiment puis à étudier les variations de taille induites, au cours du temps, dans plusieurs autres compartiments. Cette approche permet de déterminer si l'élément tracé transite successivement dans les différents compartiments au cours du temps comme cela serait le cas dans un système linéaire, ou bien si l'élément tracé se répartit simultanément dans la majorité des compartiments. Un apport d'ions phosphate à une terre est la technique la plus simple et la plus rapide pour modifier la quantité d'ions isotopiquement échangeables d'un système. La démarche expérimentale consistera alors 
à comparer les cinétiques d'échanges isotopiques observées dans des systèmes sol-solution ayant reçu des quantités variables d'ions phosphate qui seront restées en contact avec des terres pendant des durées plus ou moins longues. Les quantités choisies pour les apports ont été de 0,30 et $100 \mathrm{mgP}$. $(\mathrm{kg} \mathrm{sol})^{-1}$. Les durées de contact entre le phosphore apporté et le système sol-solution ont été de $30 \mathrm{~min}$, $3 \mathrm{~h} 30 \mathrm{~min}$ et $24 \mathrm{~h}$ avant l'injection des ions ${ }^{32} \mathrm{PO}_{4}$. Une cinétique d'échange n'est aisément analysable que durant des périodes où le système peut être considéré comme étant en état stationnaire. Or durant les premières $\mathrm{h}$ qui suivent leur addition au mélange, une partie des ions ajoutés se transfèrent vers la phase solide du sol (Barrow and Shaw, 1975). Pour les durées de contact entre l'ajout et le sol les plus faibles, une cinétique d'adsorption pouvait s'additionner à la cinétique d'échange et la cinétique globale devenir délicate à interpréter. C'est pourquoi la cinétique d'échange destinée à caractériser l'état des ions phosphates dans le système sol-solution pour lequel la durée de contact entre l'apport et le sol a été de 30 min avant l'addition du traceur a été établie pour des prélèvements et filtrations réalisés soit à $1,2,3$ et $5 \mathrm{~min}$; pour la durée de contact égale à $3 \mathrm{~h} 30 \mathrm{~min}$ les prélèvements ont eu lieu à $1,3,7$ et $10 \mathrm{~min}$ après l'addition du traceur. Durant ces 5 ou $10 \mathrm{~min}$, la vitesse d'évolution du système est suffisamment faible au regard des vitesses observées pour les cinétiques d'échanges pour que l'on puisse considérer le système comme étant en état stationnaire. Ce type d'expérience a été effectué sur plusieurs dizaines de terres représentatives des types rencontrés dans la pratique agricole.

\section{Échantillons de terre}

L'échantillon utilisé pour étudier l'organisation spatiale des différents compartiments du phosphore assimilable provient d'une terre argilo-calcaire prélevée à Saint-Vivien-de-Médoc (33590-Gironde). Son $\mathrm{pH}$-eau est de 8,1 ; il contient $48 \%$ d'argile, $2,3 \%$ de calcaire total, $1,6 \%$ de carbone organique et $93 \mathrm{mgP}$ $\mathrm{kg}^{-1}$ extrait selon la méthode Joret-Hébert. Cet horizon de surface est représentatif de la majorité des parcelles cultivées dans le Médoc en bordure de Gironde.

Des échantillons prélevés dans 2 dispositifs d'expérimentation de longue durée permettent d'illustrer les effets à long terme des pratiques de fertilisation. Les premiers proviennent du dispositif Dehérain, implanté en sol calcaire $\left(9,7 \% \mathrm{CaCO}_{3}\right)$ et situé à Grignon (Morel, 1976) : ils permettent d'apprécier l'effet de prélèvements continus sans restitution. Les seconds sont destinés à illustrer l'effet d'apports de phosphore supérieurs aux exportations des récoltes; ils ont été prélevés en sol neutre dans le dispositif dit des "48 parcelles" implanté par G Barbier à Versailles dans le domaine de la station centrale de I'INRA (Boniface et Trocmé, 1988). Cet essai comportait 8 traite- ments correspondant à des apports, cumulés sur 4 ans au moment du prélèvement, de $0,48,109,218$, 283, 331, 393 et $502 \mathrm{kgP} . \mathrm{ha}^{-1}$ effectués sous forme de superphosphate triple.

\section{RÉSULTATS ET DISCUSSION}

\section{Validité des équations représentatives des cinétiques d'échange}

Ni la période du 32P, ni celle du 33P, ne permettent de conduire des expériences au-delà de 6 mois (Morel et Fardeau, 1991). L'extrapolation au-delà de 6 mois des résultats présentés ne peut que faire l'objet d'hypothèses. Certes la formule [1] indique que l'équilibre isotopique ne peut être atteint que pour un temps infini. Cependant en supposant que la cinétique d'échange conserve la loi simplifiée [2], la formule [4] permet d'estimer le temps nécessaire pour que la totalité du phosphore minéral d'un échantillon soit isotopiquement échangé. En prenant comme exemple un échantillon précédemment décrit (Fardeau et al, 1991), pour lequel le phosphore total $P_{T}=850 \mathrm{mgP}(\mathrm{kg} \mathrm{sol})^{-1}$, le phosphore minéral $P_{M T}=660 \mathrm{mgP}(\mathrm{kg} \mathrm{sol})^{-1}$, $E_{\text {Pie1 }}=11,5 \mathrm{mgP}(\mathrm{kg} \mathrm{sol})^{-1}$ et $n=0,25$, le temps pour obtenir l'équilibre serait tel que : $660=$ $11,5 t^{0,25}$ soit $t=10^{7,03} \mathrm{~min}$ soit une durée de 21 ans. Cette valeur est suffisamment élevée pour sembler vraisemblable, sans toutefois pouvoir être considérée comme exacte puisque la valeur du terme correctif $r_{\text {eq }} / R$ contribuera à accroître significativement cette durée. C'est pourquoi l'extrapolation des résultats à une durée d'1 an, c'est-à-dire à celle d'un cycle cultural, peut être admise sans toutefois être démontrée stricto sensu dans le cas du phosphore. Par contre l'utilisation du $40 \mathrm{~K}$ dont la période est de 1,3 $10^{9}$ années a permis de vérifier cette relation dans le cas du potassium pour une durée de culture légèrement supérieure à l'année (Fardeau et al, 1992). Extrapoler des résultats sur des durées relativement longues pose le problème de la précision des différentes déterminations. Les coefficients de variation observés pour un échantillon donné sont de $5 \%$ pour $C_{P}, 3 \%$ pour $r_{1} / R, 2 \%$ pour $n$ (Fardeau, 1981); ils sont de $10 \%$ pour $C_{P}, 5 \%$ pour $\mathrm{r} 1 / \mathrm{R}, 3 \%$ pour $n, 15 \%$ pour $K_{m}$ et $F_{m}$ pour des échantillons provenant de plusieurs parcelles ayant reçu le même traitement agronomique dans un dispositif expérimental de plein champ (Oberson et al, 1993). 


\section{Modèle compartimental : analyse mathématique et choix du nombre de pools}

Un des pools, identifié de longue date (Fardeau et Marini, 1968), est constitué pour partie de la totalité des ions phosphate présents dans la solution et pour partie d'ions phosphate associés à la phase solide; ces ions ont la propriété de passer de la phase solide à la phase liquide du sol en un temps inférieur à $15 \mathrm{~s}$, durée minimale nécessaire pour effectuer une injection d'ions ${ }^{32} \mathrm{PO}_{4}$ puis un prélèvement et une filtration. Tous les ions de ce compartiment ont la même probabilité de prélèvement par les plantes : ils constituent le pool $P_{L}$ des ions libres qui participent à la nutrition des plantes sans qu'ils aient à subir de transformation chimique. La quantité de phosphore présente dans ce pool ne peut être déterminée avec rigueur et précision que par une technique de double dilution isotopique (Fardeau et Marini, 1968). Mais cette détermination consomme du temps et conduit à un lot important de vaisselle radioactive. C'est pourquoi pour la majorité des échantillons de terre la valeur retenue pour la quantité de phosphore contenu dans $P_{L}$ sera la quantité $E_{P i e 1}$. Ce n'est que dans le cas des sols à très fort pouvoir fixateur que la quantité $E_{P i e 1}$ surestime de $10 \%$ à $20 \%$ la quantité de phosphore du pool des ions directement biodisponibles (Salcedo et al, 1991). Dans ce cas, une mesure par double dilution isotopique peut s'imposer.

La recherche du nombre de pools d'ions mobiles existant en plus du pool $P L$ est effectuée en décomposant la cinétique isotopique, $r_{t} / R=f$ $(t)$, en une somme d'exponentielles. Cette décomposition peut être effectuée soit graphiquement soit par calcul (Sheppard, 1962; Shipley, 1972).

La démarche graphique est la plus didactique. Elle consiste à placer les couples de points expérimentaux $\left(r_{t} / R, t\right)$ sur un graphique dont l'axe des ordonnées portera les valeurs de $\log \left(r_{t} / R\right)$ et l'axe des abscisses celles de $t$. Puis en partant des valeurs de $t$ les plus grandes, pour lesquelles on suppose avoir atteint pour $r_{t} / R$ une valeur proche de la valeur d'équilibre, on aligne le maximum de points. Lorsque les points expérimentaux ne sont manifestement plus situés sur cette première droite, la même opération est entreprise avec les données qui précédaient les premières utilisées en utilisant des valeurs d'équilibre qui auraient été observées si le com- partiment le moins échangeable n'avait pas existé; et ainsi de suite. Lorsque cette opération est réalisée pour les cinétiques d'échange des ions phosphate, il apparaît que le nombre de compartiments mis en évidence croît avec le nombre des données expérimentales disponibles : toute donnée expérimentale supplémentaire obtenue entre 2 données déjà existantes impose de découper tout "ancien" compartiment en deux "nouveaux" compartiments (Fardeau, 1981). Ainsi le nombre de compartiments qu'il est possible de mettre en évidence, sur la base de l'interprétation des cinétiques d'échanges, ne semble limité que par le nombre et la précision des données expérimentales. On retrouve ainsi le fait que les constantes de vitesses d'échange, directement reliées aux pentes de ces droites successives, présentent un continuum de valeurs étalées de 0 à l'infini (Fardeau et al, 1991).

La démarche mathématique permet de décomposer les équations [1] ou [2] en une somme d'exponentielles, le choix du nombre d'exponentielles pouvant théoriquement être largement facilité par l'utilisation de méthodes statistiques (Wallach and Goffinet, 1987, 1989). La qualité acceptée de la précision limite le nombre d'exponentielles. Cependant les constats déduits de cette démarche sont à l'évidence les mêmes que ceux déduits de l'examen graphique des cinétiques. Les conclusions sont alors que :

- chaque ion phosphate échangeable, puisqu'il possède une constante de vitesse d'échange individualisée, devrait être considéré comme un pool stricto sensu,

- le nombre de pools "phosphate biodisponible" devrait être alors considéré comme infini. Sous cette forme, ces résultats ne semblent ni exploitables directement pour interpréter les variations des situations de nutrition phosphatée dans les sols en place, ni schématisables aisément.

Puisque :

- un compartiment est défini à partir de l'homogénéité de ses caractéristiques cinétiques d'échange avec les compartiments voisins,

- un continuum de constantes de vitesse d'échange existe, toute quantité d'ions phosphate échangeables entre 2 instants $t_{n}$ et $t_{n+1}$ peut alors être considérée comme un compartiment possédant une caractéristique cinétique homogène; en effet la caractéristique cinétique moyenne de la quantité échangeable entre $t_{n}$ et $t_{n+1}$ sera significativement différente de celle de toute autre quantité échangeable dans un espace de temps différent de $t_{n}$ à $t_{n+1}$. Ainsi le 
nombre de compartiments «phosphate biodisponible" devient dépendant des intervalles de temps choisis.

\section{Organisation spatiale des compartiments}

La lourde tradition, en chimie du sol, des extractions séquentielles du type Chang et Jackson (1956) aussi bien que l'électro-ultrafiltration ( $\mathrm{Ne}$ meth, 1985) conduisent tout un chacun à imaginer des modèles linéaires pour représenter le phosphore mobile des sols. Ainsi tout ion phosphate libre ajouté à un sol passerait d'abord dans un compartiment où il serait un peu moins mobile, puis dans une succession d'autres où il deviendrait encore moins mobile; le passage à un état donné ne pourrait avoir pour origine qu'un état légèrement plus mobile ou légèrement moins mobile.

La structure du système pluricompartimental est déduite de l'analyse des modifications de la taille de compartiments provoquées par une, ou plusieurs, additions d'ions phosphate à un échantillon de terre. Les paramètres des cinétiques d'échanges isotopiques des ions phosphate des différents traitements réalisés sur l'échantillon de terre du Médoc sont regroupés dans le tableau II. Ils permettent, au moyen des formules [3] ou [4], le calcul de la quantité de phosphore contenue dans tout compartiment défini par n'importe quel espace de temps, ainsi que les valeurs des constantes de vitesse d'échange, $K_{m}$, et celles des flux de transfert, $F_{m}$, de phosphore entre phase liquide et phase solide (Fardeau et al, 1991).

Les compartiments choisis ici sont ceux correspondant aux intervalles de temps utilisés pour des expériences d'échanges isotopiques. II s'agit donc de $E_{p i e 1}$, de $\left(E_{p i e 3^{-}} E_{p i e 1}\right)$, de $\left(E_{P_{i e 7^{-}}}\right.$ $\left.E_{\text {Pie3 }}\right)$, de $\left(E_{P i e 10}-E_{P i e 7}\right)$ à et de $\left(E_{P i e 10}-E_{P i e 1}\right)$. Les quantités de phosphore présentes dans ces divers compartiments ont été regroupées dans le tableau III. Les résultats montrent que, pour un apport donné, la quantité $E_{p i e 1}$ décroît lorsque le temps de contact entre l'apport et les particules de sol croît mais que les quantités présentes dans un compartiment donné, à l'exception de $E_{\text {pie1 }}$, ne varient pas de manière significative. Si l'ensemble pluricompartimental des ions phosphate biodisponibles était organisé selon un modèle linéaire, les ions quittant $E_{P i e 1}$ devraient passer successivement dans les différents pools, des plus mobiles au moins mobiles: la diminution de $E_{P i e 1}$ devrait induire prioritairement un accroissement du pool $\left(E_{P i e 3}-E_{P i e 1}\right)$, puis celui-ci devrait décroître au cours du temps au bénéfice de $\left(E_{P i e 7}-E_{P i e 3}\right)$, et ainsi de suite. Les données du tableau 111 montrent que cette hypothèse n'est pas vérifiée, la quantité présente dans chaque compartiment ne variant pas de manière significative au cours du temps. Ce résultats est indépendant d'une part du pas de temps retenu pour les durées de contact entre apport et sol et, d'autre part, du pas de temps choisi pour définir les différents compartiments, pour autant que les déterminations soient réalisées dans des conditions d'état pseudostationnaire. Ces résultats illustrent que dès les premiers instants du contact entre l'apport et les particules de terre, le pool des ions phosphate libres «distribue» des ions phosphate dans tous les compartiments, y compris dans ceux contenant les ions les moins mobiles. Ils confirment des informations obtenues par différents chercheurs ayant ajouté des ions phosphate mar-

Tableau II. Paramètres caractéristiques des cinétiques d'échanges isotopiques dans un échantillon ayant reçu plusieurs doses de phosphore.

\begin{tabular}{|c|c|c|c|c|c|c|c|}
\hline $\begin{array}{l}\text { Apport } \\
\text { mgP.kg-1 }\end{array}$ & $\begin{array}{l}\text { Durée de contact } \\
\qquad \text { min }\end{array}$ & $\begin{array}{c}C_{P} \\
m g P . \digamma^{\prime}\end{array}$ & $r_{1} / R$ & $n$ & $\begin{array}{c}E_{\mathrm{Pie} 1} \\
m g P . k g^{-1}\end{array}$ & $\begin{array}{c}\mathrm{K}_{\mathrm{m}} \\
\mathrm{min}^{-1}\end{array}$ & $\underset{m g P K g^{-1} m^{-1}}{\mathrm{~F}_{\mathrm{m}}}$ \\
\hline \multirow[t]{2}{*}{0} & 1 & 0,10 & 0,14 & 0,27 & 7,3 & 411 & 427 \\
\hline & 30 & 0,66 & 0,27 & 0,20 & 24,0 & 127 & 843 \\
\hline \multirow[t]{3}{*}{30} & 210 & 0,44 & 0,24 & 0,23 & 18,3 & 113 & 501 \\
\hline & 1440 & 0,28 & 0,20 & 0,26 & 14,4 & 126 & 355 \\
\hline & 30 & 3,4 & 0,41 & 0,097 & 82 & 1036 & 35000 \\
\hline \multirow[t]{2}{*}{100} & 210 & 2,6 & 0,39 & 0,13 & 66 & 181 & 4720 \\
\hline & 1440 & 1,3 & 0,31 & 0,19 & 40 & 103 & 1308 \\
\hline
\end{tabular}


Tableau III. Influence de la quantité de phosphore apportée et du temps de contact entre apport et terre sur la taille des compartiments de phosphore isotopiquement échangeable ( $E_{\text {Piet }}$ : quantité de phosphore isotopiquement échangeable en un temps texprimé en min).

Traitement

$\begin{array}{lc}\text { Apport } & \text { Durée de contact } \\ m g \mathrm{P} \mathrm{kg}^{-1} & (\mathrm{~min})\end{array}$
$m g \mathrm{P} \mathrm{kg}^{-1}$ (min)
Compartiments

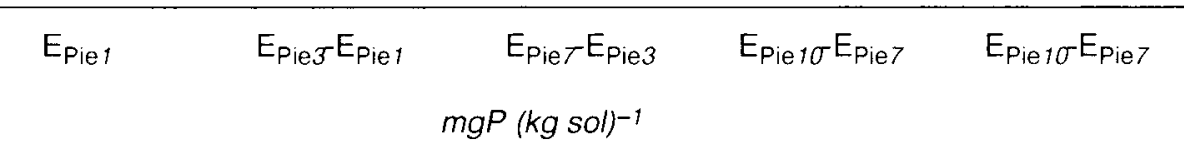

\begin{tabular}{rrrrrrr}
\hline 0 & & & & & & \\
& & & & & & \\
30 & & 2,9 & & & \\
30 & 30 & 24,0 & 5,5 & 5,0 & 2,5 & 13,0 \\
& 210 & 18,3 & 5,0 & 5,0 & 3,0 & 13,0 \\
& 1440 & 14,4 & 4,8 & 4,8 & 2,5 & 12,0 \\
100 & & & & & \\
& 30 & 82 & 9,0 & 7,0 & 4,0 & 21,0 \\
& 210 & 66 & 10,0 & 9,0 & 3,0 & 23,0 \\
& 1440 & 40 & 9,0 & 8,0 & 4,0 & 22,0 \\
\hline
\end{tabular}

qués à un sol puis ayant pratiqué des extractions séquentielles (Tandon and Kurtz, 1968; Gachon, 1972). L'information originale apportée par les résultats actuels est que des transferts vers des formes très peu mobiles ont eu lieu dès les premiers instants, information impossible à obtenir avec les extractions séquentielles beaucoup plus longues. Ces résultats illustrent également que l'analyse cinétique des variations subies par le pool E $E_{\text {Piet }}$ à l'issue d'un apport devrait permettre de prévoir, en faisant appel à des données plus élaborées que la seule concentration du phosphore dans la solution du sol (Barrow and Shaw, 1975; Barrow, 1980), la durée probable de l'effet d'un engrais phosphaté.

Cette démarche appliquée à quelques dizaines d'échantillons de terre conduit toujours à la même conclusion. Le phosphore biodisponible du sol est donc organisé majoritairement selon un modèle mamellaire (Sheppart, 1962). Le pool $P_{L}$ est le pool central du système : il «irrigue» tous les autres pools et il peut, en retour, être "irrigué" par tous les autres pools.

\section{Nombre de pools et nutrition phosphatée des plantes en place}

L'utilisation de critères physiques ne permet pas de définir un nombre fini de pools de "phosphore biodisponible». Néanmoins, les résultats présentés démontrent que le phosphore biodisponible est un ensemble très hétérogène dont les ions mobiles constitutifs n'ont pas, à un instant donné, la même probabilité de rejoindre la solution. Ils n'ont donc pas la même probabilité de participer à la nutrition des plantes. Ainsi des ions qui atteindront la solution alors qu'une racine n'est plus en mesure de les prélever doivent être considérés comme inassimilables pour cette racine. C'est donc sur la base de considérations relatives au fonctionnement des racines et du système racinaire, voie de passage obligée du phosphore en provenance du sol, qu'il semble possible de définir le nombre et l'amplitude des intervalles de temps susceptibles de caractériser des pools fonctionnels.

Chaque racine comporte au voisinage de la coiffe une zone dans laquelle a lieu un processus actif de prélèvement des ions phosphate, comme des ions potassium d'ailleurs. La durée de fonctionnement de cette zone de transfert actif est de 1-2 j (Barber, 1984). Passée cette période le prélèvement dans cette zone de la racine devient passif et beaucoup plus limité en quantité (Nelson, 1968). L'existence de zones de prélèvement actif dépend donc de la vitesse de croissance du système racinaire. Cette analyse des conditions de fonctionnement du système racinaire est confirmée par les observations suivantes : les quantités de phosphore et de potassium prélevées par une plante sont directement reliées à la vitesse d'accroissement du système racinaire (Bosc et Maertens, 1981) et non à sa longueur. Ainsi tout arrêt de croissance du système racinaire, provoquée par exemple 
par une mauvaise structure physique du sol, entraîne un arrêt rapide des prélèvements. C'est pourquoi, en un site donné du sol, la période de contact entre une zone racinaire de prélèvement actif doit être considérée comme une donnée essentielle, les ions phosphate migrant très peu. Par contre, au niveau d'un sol, la période à considérer sera celle durant laquelle un système racinaire est susceptible de posséder, du fait de son accroissement, des zones de prélèvement actif. L'analyse des courbes de prélèvement des phosphates par une culture illustre que cette période n'est pas supérieure à 3 mois dans le cas des cultures annuelles.

Ces données écophysiologiques, et l'existence du pool $P_{L}$ des ions libres physiquement défini, autorisent donc à proposer de découper en 5 compartiments l'ensemble des ions phosphate isotopiquement échangeables. Le premier compartiment, central dans le système, est celui des ions immédiatement disponibles : il contient la quantité $P_{L}$ non significativement différente de la quantité $E_{\text {Pie1 }}$ pour la majorité des échantillons de terre (Salcedo et al, 1991). Le compartiment B est celui des ions échangeables entre $1 \mathrm{~min}$ et $1 \mathrm{j}$ (1 $440 \mathrm{~min}$ ), 1 j étant la durée moyenne de prélèvement actif d'une zone racinaire : il contient la quantité $E_{B}=\left(E_{1440}-E_{P i e 1}\right)$. Le compartiment $C$ est celui des ions échangeables entre $1 \mathrm{j}$ et 3 mois, 3 mois étant la durée moyenne d'accroissement d'un système racinaire : il contient la quantité $E_{C}=\left(E_{129600}-E_{1440}\right)$. Le compartiment $D$ est celui des ions échangeables entre 3 mois et 1 an, 1 an correspondant à un cycle cultural annuel : il contient la quantité $E_{D}=\left(E_{518} 400\right.$ - $E_{129800}$ ). Le compartiment $F$ correspond à des ions échangeables en un temps supérieur à 1 an : il contient au maximum la quantité $E_{F}=$ (PMT $-E_{518400}$ ).

\section{Représentation des différents compartiments du modèle fonctionnel du phosphore biodisponible}

Pour être en mesure de visualiser le phosphore biodisponible présent dans des échantillons de terre, il était nécessaire d'adopter une représentation symbolisée du modèle. La représentation devait simultanément sembler simple et contenir un maximum d'informations schématisées à l'extrême. Elle devait en particulier rendre compte visuellement des 3 facteurs considérés comme indispensables pour caractériser de façon satisfaisante le phosphore biodisponible (Becket and White, 1964; Dalal and Hallsworth, 1977); il s'agit des facteurs quantité, intensité et capacité, c'est-à-dire du pouvoir tampon du sol vis-à-vis des ions phosphate (Holford, 1979, 1991; Moody, 1991).

Tous les pools ont été schématisés par des rectangles dont les surfaces sont proportionnelles aux quantités de phosphore qu'ils contiennent; la surface de chaque rectangle symbolise donc, pour chaque pool, leur facteur quantité.

\section{Représentation du pool $P_{L}$ des ions libres}

La hauteur du rectangle représentatif de $P_{L}$ est proportionnelle à la valeur de la concentration, $C_{P}$, généralement considérée comme le facteur intensité des ions phosphate dans la solution du sol (Beckett and White, 1964). Cette hauteur représentative de $C_{P}$, facteur déterminant des quantités de phosphore prélevées dans une solution nutritive (Barber, 1984), symbolise donc le facteur intensité. La surface du rectangle étant proportionnelle à la quantité $P_{L}$, sa longueur est proportionnelle au rapport $P_{L} / C_{P}$, c'est-à-dire au rapport du facteur quantité au facteur intensité. Le rapport $P_{L} / C_{P}$ est donc exactement le facteur capacité. La longueur représentative du pool $P_{L}$ symbolise donc le facteur capacité. Ce rapport a la dimension d'un volume; il s'agit d'un volume apparent de solution de sol qui représente le volume de solution que contiendrait le sol en faisant l'hypothèse que cette solution aurait la même concentration d'ions phosphate que la solution du sol. II s'agit en fait du volume de solution que le sol peut fournir sans que la concentration du phosphore dans cette solution varie. Puisque $P_{L}$ n'est que très rarement significativement différent de $E_{P i e 1}$ (Salcedo et al, 1991) et que $E_{P i e 1}=10 C_{P}$ $(R / r 1)$, la longueur $P_{L} / C_{P}$ du pool $P_{L}$ est proportionnelle à la quantité $10(R / r 1)$. Les valeurs de $R / r 1$ étant corrélées au pouvoir fixateur du sol vis-à-vis des ions phosphate (Sen Tran et al, 1988; Morel et Fardeau, 1989), ces résultats permettent de conclure que pouvoir tampon et pouvoir fixateur d'un sol vis-à-vis des ions phosphate sont liés. La structure du rectangle représentatif de $P_{L}$, nœud du modèle compartimental, illustre donc les facteurs généralement recherchés pour caractériser le phosphore biodisponible. 


\section{Représentation des autres pools du phosphore biodisponible}

Les connaissances sur les pools B, C, D et $F$ sont beaucoup plus limitées que celles acquises sur $P_{L}$. Elles sont réduites au contenu des compartiments et au fait que ces 4 compartiments sont branchés sur $P_{L}$. En particulier il n'est pas actuellement possible de préciser la valeur du facteur intensité pour chacun des pools, c'est-àdire la valeur que prendrait la concentration des ions phosphate si le pool $P_{L}$ avait été seul retiré, par exemple, sous l'effet d'un prélèvement racinaire. C'est pourquoi nous avons arbitrairement attribué à ces pools une hauteur unique indépendante de l'échantillon analysé. Dans ces conditions leur longueur est fonction de leur surface, c'est-à-dire de leur contenu. Comme le feront apparaître les exemples qui suivront, la quantité de phosphore contenue dans le pool $P_{L}$ est toujours très inférieure à celles présentes dans les autres pools. Cependant vis-à-vis de la satisfaction instantanée des besoins des cultures, $P_{L}$ présente un intérêt supérieur à celui des autres pools puisque ses ions sont assimilables sans transformation chimique. Afin de pouvoir présenter sur une feuille unique les 5 pools, tout en insistant sur le rôle prépondérant de $P_{L}$, l'échelle retenue pour la surface de $B, C, D$ et $F$ a été réduite par un facteur $3 / 25$, le choix de ce facteur n'étant dû qu'à la simplicité qu'il entraîne dans l'utilisation de papier graphique. L'existence du continuum de constantes de vitesses de transfert et l'impossibilité actuelle de démontrer que les transferts entre $B, C, D$ et $F$ passent obligatoirement par $P_{L}$ ne peuvent pas encore nous autoriser à schématiser ces pools en utilisant une séparation franchement matérialisée entre chacun d'entre eux. Seuls leurs contours seront représentés. Cependant il semble bien que les changements de formes chimiques et physiques des phosphates ne puissent pas avoir lieu sans passage par la forme ionique libre. La figure 1 contribue à synthétiser et schématiser l'ensemble du modèle.

\section{Apport du modèle à la connaissance du P biodisponible}

Le schéma illustre d'abord le pouvoir tampon «instantané» du sol; il est fonction de la structure du pool $P_{L}$ : il permet, dans la limite de la quantité $P_{L}$, de maintenir instantanément la concentra-

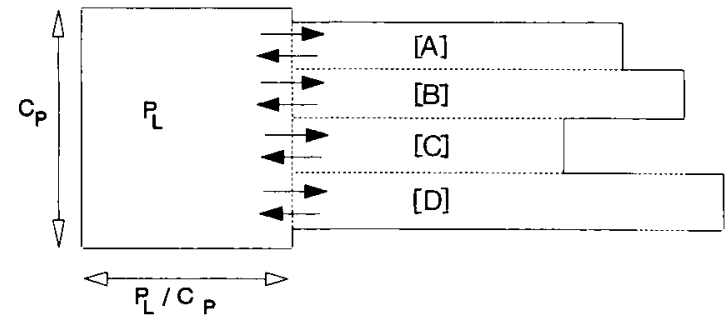

Fig 1. Représentation schématique du phosphore biodisponible $\left(C_{P}\right.$ : concentration des ions phosphate dans la solution du sol; $P L$ : compartiment échangeable instantanément; $B$ : compartiment échangeable entre 1 min et $1 \mathrm{j}$; $\mathrm{C}$ : compartiment échangeable entre $1 \mathrm{j}$ et 3 mois; $\mathrm{D}$ : compartiment échangeable entre 3 mois et 1 an; $F$ : compartiment échangeable en plus d'un an).

tion du phosphate constante dans la solution lors des prélèvements par les racines; en cas de consommation locale par une zone racinaire supérieure à la quantité $P_{L}$, le pool B prendra le relais pour assurer tout ou partie de la satisfaction des besoins des cultures.

Ce schéma démontre en plus l'existence, pressentie mais non décrite jusqu'à maintenant, d'un pouvoir tampon "retard" associé aux 4 pools dont les possibilités de transferts vers $P_{L}$ dépendent du temps. Le rôle de ces 2 pouvoirs tampons vis-à-vis de la nutrition phosphatée des cultures est différent : «l'instantané», avec intervention éventuelle du pool $B$, permet de maintenir constantes les possibilités de satisfaction instantanée des besoins des plantes, le "retard" assurera le renouvellement des potentialités nutritives des agrosystèmes à l'échelle annuelle ou pluriannuelle.

Ce schéma permet de rappeler qu'il n'est vraiment plus possible de considérer qu'une terre contiendrait côte à côte du phosphore biodisponible et du phosphore non biodisponible : une terre, quelle qu'elle soit, contient du phosphore plus ou moins rapidement biodisponible. C'est donc un "combat de cinétiques" auxquels sols et plantes se livrent pour les ions phosphate.

Ce schéma permet enfin d'expliquer l'origine de la contradiction signalée entre d'une part les déterminations de type quantité $A$ ou $L$ et d'autre part les cinétiques d'échanges isotopiques. Le phosphore biodisponible est bien constitué de plusieurs pools à potentiel nutritif distinct; mais l'existence de ces pools ne peut être révélée qu'avec l'utilisation d'outils de mesure dont la vitesse est supérieure à celle des mécanismes mis en jeu entre les différents pools. Dans un sol en 
place la plante, outil de prélèvement de phosphore du sol, ne peut rendre compte de mécanismes dont les durées sont de l'ordre de la minute ou de la journée; elle peut au mieux révéler des phénomènes dont la durée est du même ordre de grandeur que le temps de culture, c'està-dire 3 à 4 semaines. La plante n'était donc en aucun cas l'outil à utiliser pour mettre en évidence l'existence de pools «phosphore biodisponible" à temps de résidence bref. Le même type de conclusion doit être transposée aux démarches utilisant des déterminations reposant sur une unique mesure effectuée après 15 jours ou 3 semaines d'échanges (Triboï et Gachon, 1988).

\section{Application du modèle fonctionnel à l'analyse de pratiques agricoles mettant en jeu le phosphore}

Les conséquences de différentes pratiques culturales sur l'état du phosphore biodisponible d'une terre peuvent être étudiées en examinant des échantillons de terre prélevés dans n'importe quel dispositif expérimental de longue durée ayant eu pour objet l'étude de la fertilisation phosphatée.

\section{Conséquences de prélèvements répétitifs de phosphore sans restitution compensatrice des exportations}

La figure 2 regroupe les résultats des échantillons de terre du dispositif Dehérain prélevés en 1938 et en 1972 dans la parcelle $n^{\circ} 47$ cultivée en permanence sans jamais recevoir d'engrais phosphatés (Morel, 1976). La quantité de phosphore prélevée dans cette parcelle entre 1938 et 1972 a été de 100 mgP. $\mathrm{kg}^{-1}$. Ce schéma montre que les prélèvements de phosphore entraînent simultanément une diminution des facteurs inten-

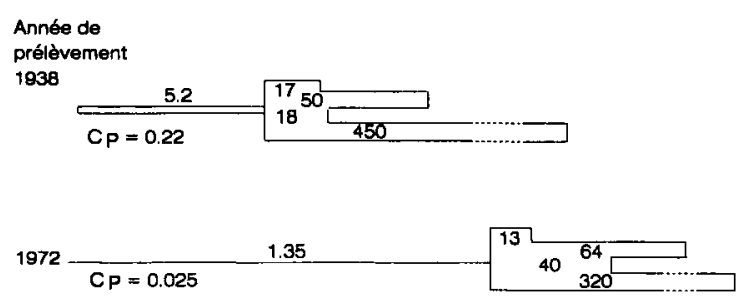

Fig 2. Influence de prélèvements répétitifs non compensés par des rapports d'engrais sur la taille des différents compartiments du phosphore biodisponible. $\left(C_{P}: \mathrm{mgP}^{-1}\right.$; quantité de $P$ dans les compartiments : $\left.\left.\mathrm{mP}(\mathrm{kg} \mathrm{sol})^{-1}\right)\right)$. sité et quantité du pool $P_{L}$ mais une augmentation simultanée du facteur capacité, donc du pouvoir fixateur. II illustre également que, sous cultures annuelles continues, des exportations nettes de phosphore induisent toujours une redistribution du phosphore entre tous les pools; des ions originaires du pool $F$, qui n'étaient pas échangeables en 1938 entre 1 j et 3 mois (Pool $C$ ), ou bien entre 3 mois et 1 an (Pool D), peuvent devenir échangeables dans ces intervalles de temps lorsque le facteur intensité décroît sous l'action des prélèvements racinaires continus. Ainsi on peut admettre que, sous prélèvement continu sans restitution, la fertilité phosphorique du sol décroît mais elle ne semble pas devoir s'annuler totalement, sauf à éliminer la totalité du phosphore.

\section{Conséquences d'apports de phosphore et de prélèvements simultanés par des cultures}

La figure 3 regroupe les données de quelques échantillons prélevés dans le dispositif INRA des «48 parcelles». Les schémas illustrent les conséquences d'apports de 0, 218 et $393 \mathrm{kgP}$.ha-1 qui correspondent à des additions de phosphore sensiblement égales à 0,65 et $118 \mathrm{mgP} . \mathrm{kg}^{-1}$. Ils permettent de constater, entre autres choses, que d'une part, dans un sol donné, les variations des paramètres intensité, quantité et capacité sont liés, et d'autre part du phosphore apporté sous forme instantanément biodisponible se répartit dans les différents compartiments : sa mobilité est réduite mais non nulle.
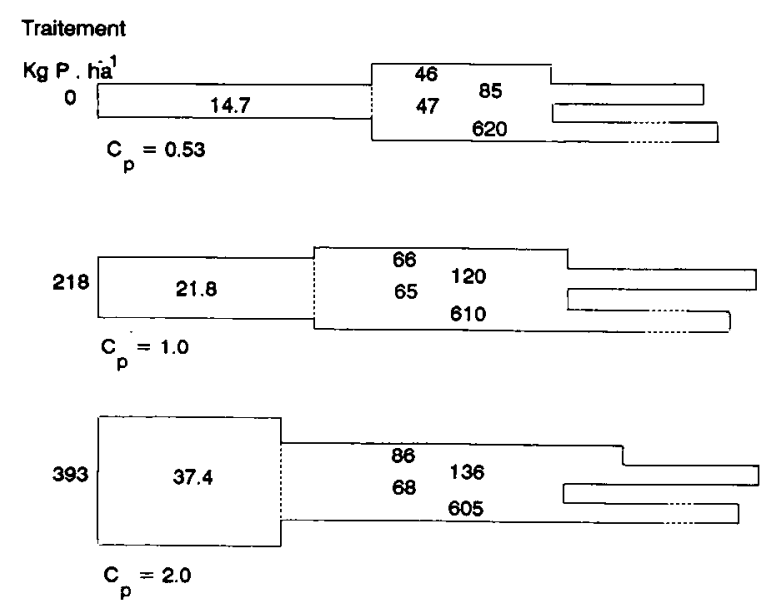

Fig 3. Influence d'apports de phosphore supérieurs aux quantités exportées par les récoltes sur la taille des différents compartiments du phosphore biodisponible $\left(C_{P}: \mathrm{mgP}\right.$ $1^{-1}$; quantité de $\mathrm{P}$ dans les compartiments : $\operatorname{mg} \mathrm{P}(\mathrm{kg} \mathrm{sol})^{-1}$. 


\section{Limites du modèle}

Les données relatives à la taille des compartiments ont été obtenues en plaçant le système analysé en état stationnaire, ou steady-state. Les valeurs ainsi calculées doivent être considérées comme des valeurs minimales. En effet lorsque les racines prélèvent du phosphore, les ions phosphate de la phase solide du sol rejoindront la solution également sous l'effet d'un processus diffusif lié au gradient de concentration créé par le prélèvement. Dans de telles circonstances la valeur de $K_{\mathrm{m}}$ et la taille de certains compartiments pourront alors être augmentées.

\section{CONCLUSION}

Les techniques d'extractions chimiques sélectives séquentielles (Chang and Jackson, 1956; Hedley et al, 1982; Frossard et al, 1989), de diffraction X (Lindsay et al, 1962), d'électroultrafiltration (Nemeth, 1985), de RMN (Condron et al, 1990) démontrent qu'il existe dans les sols de très nombreuses formes chimiques de phosphore minéral et organique (Anderson, 1980; Cosgrove, 1980). La synthèse de ces connaissances permet de concevoir des modèles représentant le phosphore du sol; certains sont particulièrement élaborés (Halm et al, 1972; Chauchan et al, 1981). L'intérêt de ces modèles est cependant limité lorsque l'objectif est de prévoir, pour les modifier éventuellement, les conditions de la nutrition phosphatée des cultures (Olsen et Khasawneh, 1980). En effet, d'une part ces modèles comportent bien des flèches reliant certains compartiments, mais aucune ne semble quantifiée et, d'autre part, il n'existe pas de mécanismes démontrés entre le phosphore présent dans des compartiments déterminés par voie analytique et son aptitude à satisfaire la demande des plantes. Tous ces résultats obligeaient néanmoins à admettre que le phosphore biodisponible d'un sol pouvait être, à l'image de son phosphore total, un système à plusieurs compartiments.

L'utilisation des méthodes d'analyse compartimentale faisant appel à des techniques isotopiques permet de démontrer que le phosphore biodisponible est effectivement un système à plusieurs compartiments. Le modèle fonctionnel à plusieurs pools a été déduit des connaissances écophysiologiques sur le fonctionnement des racines et des systèmes racinaires. Le mo- dèle comporte 1 pool central dans lequel les racines prélèvent le phosphore qu'elles consomment et 4 compartiments caractérisés par leurs vitesses moyennes de transfert potentiel vers le pool central.

Ce modèle semble être le seul qui permette d'expliquer l'ensemble des observations concernant les variations de biodisponibilité du phosphore présent dans le sol ou les engrais. II rend compte de l'existence du pouvoir tampon “instantané» du sol pour les ions phosphates; mais il a aussi permis de démontrer l'existence d'un pouvoir tampon "retard". II condamne le concept manichéen implicitement utilisé chaque fois qu'une méthode d'extraction est mise en œuvre pour apprécier le phosphore biodisponible. Ce dernier résultat permet de comprendre pourquoi l'utilisation des méthodes d'extractions chimiques peut parfois conduire à des approximations grossières en matière de conseils de fertilisation phosphatée des cultures (Fardeau et Frossard, 1991). C'est ainsi, par exemple, que leur utilisation a favorisé l'épanouissement de la notion de "vieille graisse" qu'il n'était possible de constituer que par des apports très supérieurs aux exportations. Cette notion est maintenant entièrement remise en cause (Gachon, 1988).

On peut reprocher au modèle proposé de ne pas prendre en considération les variations de biodisponibilité du phosphore organique. Le phosphore organique stricto sensu, tel que celui des phytates de calcium, de magnésium, de fer ou d'aluminium, n'est pas isotopiquement échangeable. La contribution de ces composés à la nutrition des cultures suppose leur minéralisation préalable; celle-ci dépend à la fois de la cinétique de minéralisation des composés carbonés récents (Tiessen, 1991) et de la présence de phosphatases actives (Oberson et al, 1993). Des bilans nets pluri-annuels de phosphore organique, effectués dans quelques parcelles expérimentales, laissent à penser que, pour la pratique agricole en vigueur dans les sols de l'Ouest européen portant de très longue date des cultures annuelles, le rôle du phosphore organique serait très peu important au regard de celui joué par les fractions minérales échangeables. Il est évident que la situation est radicalement différente dans des écosystèmes tels que les prairies permanentes pour lesquelles la minéralisation d'une faible fraction du phosphore organique peut suffire à alimenter les plantes.

L'extension de ce type de modèle à d'autres éléments biodisponibles dépend de leur dynami- 
que dans les sols. Elle est envisageable chaque fois que la forme échangeable de l'élément sera la source nutritive principale pour les plantes et que cette forme possède une cinétique d'échange iso-ionique dans des systèmes solsolution. C'est dire qu'en dehors de l'azote et du soufre, dont les destins sont majoritairement liés à l'activité biologique des sols, ce type de modèle semble utilisable pour apprécier la biodisponibilité pour les plantes de beaucoup d'éléments minéraux. En effet, des cinétiques d'échanges isoioniques entre phase liquide et phase solide du sol existent pour de très nombreux éléments (Fardeau et Guiraud, 1974) : il s'agit aussi bien d'éléments indispensables à la vie, tels que le potassium ou le calcium, que des éléments nocifs tels que le cadmium ou le mercure.

\section{RÉFÉRENCES}

Anderson G (1980) Assessing organic phosphorus in soils. In: The role of phosphorus in agriculture (FE Khasawneh, EC Sample, EJ Kamprath, eds) Am Soc Agron, Madison, WI, 361-410

Atkins GL (1973) Modèles à compartiments multiples pour les sytèmes biologiques. Gauthiers-Villars, Paris

Barber SA (1984) Soil nutrient bioavailability. A mechanistic approach. John Wiley, New York

Barbier G, Tyszkiewicz E (1952) Mobilité des ions phosphoriques fixés dans le sol, étudiée au moyen de 32P. Assoc Int Sci Sol, Commission I et IV, Dublin, 79-81

Barbier G, Tyszkiewicz E (1954) Extension, par dessication, de l'autodiffusion des ions phosphoriques dans le sol. CR Séances Acad Sci Paris 238, 19, 1908-1910

Barrow NJ (1980) Evaluation and utilization of residual phosphate in soils. In: The role of phosphorus in agriculture (FE Khasawneh, EC Sample, EJ Kamprath, eds) Am Soc Agron, Madison, WI, 333359

Barrow NJ, Shaw TC (1975) The slow reactions between soil and anions. 2. Effect of time and temperature on the decrease in phosphate concentration in the soil solution. Soil Sci 119, 167-177

Beckett PHT, White RE (1964) Studies on the potentials of soils. Part III. The pool of labile inorganic phosphate. Plant Soil 21, 3, 253-282

Boniface R, Trocmé S (1988) Enseignements fournis par des essais de longue durée sur la fumure phosphatée et potassique. 2. Essais sur la fumure phosphatée. In: Phosphore et potassium dans les relations sol-plante : conséquences sur la fertilisation (L Gachon, ed) INRA, Paris, 279-402
Bosc M, Maertens M (1981) Rôle de l'accroissement du système racinaire dans l'absorption de divers états du potassium du sol. Agrochimica 25, 1-8

Chang SC, Jackson ML (1956) Fractionation of soil phosphorus. Soil Sci 84, 133-144

Chauchan BS, Stewart JWB, Paul EA (1981) Effect of labile inorganic phosphate status and organic carbon additions on the microbial uptake of phosphorus in soils. Can J Soil 61, 373-385

Condron LM, Frossard E, Tiessen H, Newman RH, Stewart JWB (1990) Chemical nature of organic phosphorus in cultivated and uncultivated soils under different environmental conditions. $J$ Soil $S C i$ $41,41-50$

Cosgrove DJ (1980) Inositol phosphates. Elsevier, Amsterdam

Dalal RC, Hallsworth EG (1977) Measurement of isotopic exchangeable soil phosphorus and interrelationship among parameters of quantity, intensity and capacity factors. Soil Sci Soc Am J 41 , $81-86$

Daubeny $D$ (1845) On the distinction between the dormant and active ingredients of the soil. $J R$ Agric Soc England 7, 237-245

Fardeau JC (1981) Cinétiques de dilution isotopique et phosphore assimilable des sols. Thèse, Paris VI

Fardeau JC, Frossard E (1991) Processus de transformations du phosphore dans les sols de l'Afrique de l'Ouest semi-aride : application au phosphore assimilable. In: Phosphorus cycles in terrestrial and aquatic ecosystems. Regional workshop 4: Africa (H Tiessen, E Frossard, eds) Univ Saskatchewan, Saskatoon, 108-128

Fardeau JC, Guiraud G (1974) Utilisation des filtres millipores en chimie du sol : application à la caractérisation des cinétiques de dilution isotopique des ions dans le sol. Ann Agron 25, 113-117

Fardeau JC, Jappé J (1976) Nouvelle méthode de détermination du phosphore assimilable par les plantes : extrapolation des cinétiques de dilution isotopique. CR Séances Acad Sci Paris 280, D, 1861-1864

Fardeau JC, Marini P (1968) Détermination par échange isotopique en retour, du compartiment des ions phosphate les plus mobiles. CR Acad $\mathrm{SCl}$ Paris 267, D, 324-327

Fardeau JC, Morel C, Boniface R (1988) Pourquoi choisir la méthode Olsen pour estimer le phosphore "assimilable" des sols. agronomie 8, 577-584

Fardeau JC, Morel C, Boniface R (1991) Cinétiques de transfert des ions phosphate du sol vers la solution du sol : paramètres caractéristiques. agronomie 11, 787-797

Fardeau JC, Morel C, Jappé J (1985) Cinétiques d'échanges des ions phosphate dans les systèmes sol-solution: vérification de l'équation théorique. CR Séances Acad Sci Paris 300, III, 371-376

Fardeau JC, Poss R, Saragoni H (1992) Effect of potassium fertilization on $\mathrm{K}$-cycling in different agro- 
systems. In: Potassium in ecosystems. IPI, Bâle (in press)

Fried M, Dean LA (1952) A concept concerning the measurement of available soil nutrients. Soil Sci 73, 263-271

Frossard E, Stewart JWB, St Arnaud RJ (1989) Distribution and mobility of phosphorus in grassland and forest soils of Saskatchewan. Can J Soil Sci 69, 401-416

Gachon L (1972) Fractionnement du phosphore labile en relation avec le type de sol. Ann Agron 23 (4), 429-444

Gachon L (1988) Conclusion. In: Phosphore et potassium dans les relations sol-plante: conséquences sur la fertilisation (L Gachon, ed) INRA, Paris, 557566

Gianinazzi-Pearson V, Fardeau JC, Asimi S, Gianinazzi S (1981) Source of additional phosphorus absorbed from soil by vesicular-arbuscular mycorrhizal soybeans. Physiol Veg 19, 33-43

Halm BJ, Stewart JWB, Halstead RL (1972) The phosphorus cycle in a native grassland ecosystem. In: Isotopes and radiation in soil-plant relationships including forestry. AIEA, Vienne, 571-586

Halstead RL (1967) Chemical availability of native and applied phosphorus in soils and their textural fractions. Soil Sci Soc Am Proc 31 (3), 414-419

Hedley MJ, Stewart JWB, Chauchan BS (1982) Changes in inorganic soil phosphorus fractions induced by cultivation practices and by laboratory incubations. Soil Sci Soc Am J 46, 970-976

Holford ICR (1979) Evaluation of soil phosphate buffering indices. Aust $J$ Soil Res 17, 495-504

Holford ICR (1991) Comments on intensity-quantity aspects of soil phosphorus. Aust J Soil Res 29, 13-14

Jenkins WL (1966) The relationship between "labile soil phosphorus" and the aluminium and iron-bound phosphorus in tropical soils. Plant Soil 24 (3), 407422

John MK (1970) Colorimetric determination of phosphorus in soil and plant material with ascorbic acid. Soil Sci 109, 214-220

Kotze WAG, Diest J (1973) The kinetics of the isotopic exchange of $\mathrm{P}$ and $\mathrm{Ca}$ in rock phosphate. Agrochemophysica 5, 57-60

Lamm CG (1962) Factors affecting the ratio in the plants of a nutrient derived from the fertilizer. In: Radioisotopes in soil-plant nutrition studies. STI/ PUB/55 IAFE, Vienne, 343-352

Larsen $S$ (1952) The use of $32 P$ in studies on the uptake of phosphorus by plants. Plant Soil 4, 1-10

Lindsay WL, Frazier AW, Stephenson HF (1962) Identification of reaction products from phosphate fertilizers in soils. Soil Sci Soc Am Proc 26 (5), 446452

Moody PW (1991) Reply to: "Comments on intensityquantity aspects of soil phosphorus". Aust $J$ Soil Res 29, 11-12
Morel R (1976) Utilisation des résultats obtenus sur des essais de longue durée dans l'étude des transferts de l'azote dans le sol. Ann Agron 27 (5-6), 483-486

Morel JL, Fardeau JC (1989) Pouvoir fixateur des sois vis-à-vis du phosphore : conséquence sur la fertilisation phosphatée. Perspect Agric 147, 65-72

Morel C, Fardeau JC (1991) Phosphorus bioavailability of fertilizers: a predictive laboratory method for its evaluation. Fertil Res 28, 1-9

Nelson WL (1968) Plant factors affecting potassium availability and uptake. In: The role of potassium in agriculture (VJ Kilmer, SE Younts, NC Brady, eds) ASA, Madison, WI, 355-383

Nemeth K (1985) Recent advances in EUF research (1980-1983). Plant Soil 83, 1-19

Oberson A, Fardeau JC, Besson JM, Sticher H (1993) Soil phosphorus in cropping systems managed according to conventional and biological agricultural methods. Biol Fertil Soils (in press)

Olsen SR, Khasawneh FE (1980) Use and limitations of physical-chemical criteria for assessing the status of phosphorus in soils. In: The role of phosphorus in agriculture (FE Khasawneh, EC Sample, EJ Kamprath, eds) Am Soc Agron, Madison WI, 361-410

Probert ME, Larsen S (1972) The kinetics of heterogeneous isotopic exchange. J Soil Sci 23 (1), 76-81

Salcedo IH, Bertino F, Sampaio EVSB (1991) Reactivity of phosphorus in northeastern Brazilian soils assessed by isotopic dilution. Soil Sci Soc Am J 55, 140-145

Sen Tran T, Fardeau JC, Giroux M (1988) Effects of soil properties on plant-available phosphorus determined by the isotopic dilution ${ }^{32} \mathrm{P}$ method. Soil $\mathrm{Sci}$ Soc Am J 52, 1383-1390

Sheppard CW (1962) Basic principles of the tracer theory. John Wiley and Sons, New York

Shipley RA, Clark RE (1972) Tracer methods for in vivo kinetics. Acad Press, New York

Talibudeen O (1958) Isotopically exchangeable phosphorus in soils. Part III. The fractionation of soil phosphorus. J Soil Sci 9 (1), 120-129

Tandon HLS, Kurtz LT (1968) Isotopic exchange characteristics of aluminium and iron-bound fractions of soil phosphorus. Soil Sci Soc Am Proc 32 (6), 799802

Tiessen $H$ (1991) Characterisation of soil phosphorus and its availability in different ecosystems. In: Trends in soil science. Council of scientific research integration. India, 1, 83-89

Triboï E, Gachon L (1988) Étude des méthodes d'appréciation de l'offre phosphatée du sol. I. Détermination du phosphore isotopiquement échangeable ou valeur E. In : Phosphore et potassium dans les relations sol-plante : conséquences sur la fertilisation (L Gachon, éd) INRA, Paris, 165-180

Ulrich B, Lin H, Karapurkar H (1962) Kinetics of isotopic exchange between soil phosphates, soil solution 
and plant. In: Radioisotopes in soil-plant nutrition studies. STI/PUB/55, IAEA, Vienne, 59-74

Wallach D, Goffinet B (1987) Mean squarred error of prediction in models for studying ecological and agronomic systems. Biometrics 43, 561-573
Wallach D, Goffinet B (1989) Mean squarred error of prediction as a criterion for evaluating and comparing system models. Ecol Model 44, 299-306

Wiklander L (1950) Kinetics of phosphate exchange in soils. Ann R Agric College Sweden 17, 407-424 